\title{
Imatinib Treatment of Lymphangiomatosis (Generalized Lymphatic Anomaly)
}

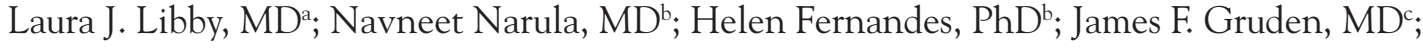
David J. Wolf, MD'; and Daniel M. Libby, MDa

\begin{abstract}
Lymphangiomatosis (eg, generalized lymphatic anomaly) is an abnormal proliferation of lymphatic endothelial cells. It is often a childhood disease, but it may present in adulthood by infiltrating organs and cause obstruction, bleeding, or disruption of lymphatic flow. Pulmonary involvement may be mild or cause diffuse interstitial lung disease, airway obstruction, hemoptysis, chylothorax, chylopericardium, and culminate in respiratory failure. Treatment has been limited to surgical resection or drainage procedures because there is no accepted effective systemic therapy. This report presents a patient with lymphangiomatosis and life-threatening hemoptysis in whom positive immunostaining for C-KIT suggested upregulation of tyrosine kinase and whose disease was controlled with imatinib.
\end{abstract}

J Natl Compr Canc Netw 2016;14(4):383-386

\section{Background}

Lymphangiomatosis (eg, generalized lymphatic anomaly [GLA]) is an abnormal proliferation of lymphatic endothelial cells. In 2014, the International Society for the Study of Vascular Anomalies (ISSVA) omitted the terms "lymphangioma" and "lymphangiomatosis" in favor of "generalized lymphatic anomaly"; the literature reflects a change in terminology. Lymphangiomatosis is often a childhood disease, but it may present in adults via infiltrating organs and cause obstruction, bleeding, or disruption of lymphatic flow. Pulmonary involvement may be mild or cause diffuse interstitial lung disease, airway obstruction, hemoptysis, chylothorax, chylopericardium, and culminate in respiratory failure. ${ }^{1}$ Treatment is limited to surgical resection or drainage procedures, ${ }^{2}$ because no approved effective systemic therapy exists. Herein, we present a case of a patient with lymphangiomatosis (GLA) and life-threatening hemoptysis for whom positive immunostaining for c-KIT suggested

From the aDepartment of Medicine, Division of Pulmonary and Critical Care Medicine, bDepartment of Pathology and Laboratory Medicine, 'Department of Radiology, Division of Body Imaging, and dDepartment of Medicine, Division of Hematology and Oncology, Joan and Sanford I. Weill Medical College of Cornell University and The New York-Presbyterian Hospital Weill Cornell Medical Center, New York, New York.

Submitted September 16, 2015; accepted for publication

February 24, 2016. upregulation of tyrosine kinase and whose disease was controlled with imatinib.

\section{Case Report}

In 2002, an asymptomatic 55-year-old woman underwent an abdominal CT as a result of an automobile accident, which showed splenomegaly. After hip trauma in 2004, CT again demonstrated splenomegaly and a new, partially cystic hepatic lesion. Results from a splenectomy and an open liver biopsy were consistent with lymphangioma. Two weeks later, she developed hemoptysis, chest pain, and hemothorax, which was managed with the placement of a chest tube for drainage. In 2005, after developing left shoulder pain, a CT showed a recurrent left hemothorax and mediastinal mass. The patient underwent decortication and evacuation of the hemothorax. In 2007, she again developed dyspnea and a left hemothorax, which was managed with the placement of a 
Libby et al

chest tube and pleurodesis. The pleural fluid cytology from all specimens was negative for malignancy.

In 2008, she developed a cough, dyspnea, and left pleuritic chest pain. Because the results of a PET/CT showed hypermetabolic right supraclavicular lymphadenopathy and diffuse left pulmonary abnormalities, a biopsy was performed of the right supraclavicular lymph node, and the results revealed lymphangioma. Sorafenib at $100 \mathrm{mg}$ orally twice daily was initiated because of its inhibitory effects on vascular endothelial growth factors (VEGFs), but the patient developed plantar-palmar skin toxicity despite dose reductions, and the drug was discontinued after 3 months due to the lack of therapeutic efficacy (persistent chest pain, dyspnea, and hemoptysis). She remained symptomatic and results of a 2008 CT demonstrated disease progression (Figure 1A). Results of pulmonary function tests showed a mild restrictive ventilatory impairment with a vital capacity of $2.37 \mathrm{~L} / \mathrm{min}$ ( $76 \%$ predicted).

During the following 3 years, she was closely followed with CT and pulmonary function tests. In 2010, sirolimus at $2 \mathrm{mg}$ orally daily (for 6 months) was prescribed because of its inhibitory effects on lymphatic endothelial cell growth, but she did not experience response. By 2011, the patient developed a severe restrictive ventilatory impairment with a vital capacity of $1.59 \mathrm{~L} / \mathrm{min}$ ( $46 \%$ predicted), and CT results (Figure 1B) showed further progression. In 2012, she developed persistent high-volume hemoptysis despite bronchial artery embolization and underwent emergency left lower lobectomy to control bleeding. Her postoperative course was complicated by hemothorax, and she returned to the operating room for evacuation of intrapleural hematoma. Based on the pathologic findings and positive immunostaining for c-KIT (Figure 2B), the patient was started on imatinib mesylate, 400 $\mathrm{mg} / \mathrm{d}$ in February 2012. The dose was subsequently reduced to $400 \mathrm{mg}$ every other day because of drug-associated diarrhea. Chest pain, dyspnea, and hemoptysis resolved, and at the time of writing her pulmonary lymphangiomatosis remained stable as evidenced by CT (Figure 1C); her vital capacity improved to 1.92 $\mathrm{L} / \mathrm{min}$ ( $59 \%$ predicted). The patient continues to receive imatinib, $400 \mathrm{mg}$ every other day, and has been clinically stable without recurrent hemoptysis for the past 4 years.
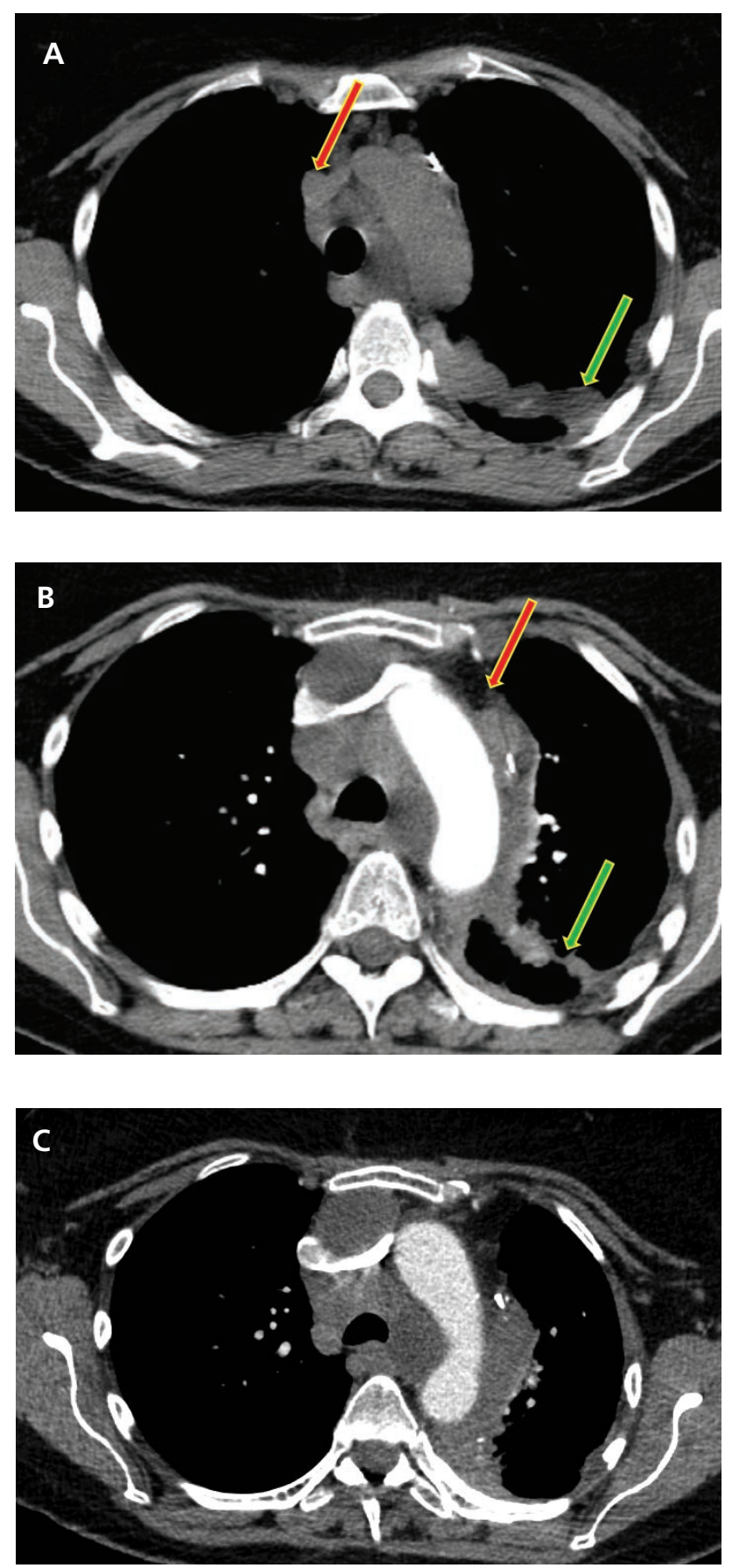

Figure 1. (A) 2008: Non-contrast-enhanced CT image through the upper chest at the level of the azygos arch shows abnormal soft tissue surrounding the trachea and deviating the superior vena cava (SVC) anteriorly and to the right (red arrow). The mass is of lower density than the chest wall musculature, as is typical with this entity. Note the pleural involvement on the left of the patient with extension into the oblique fissure (green arrow). The high-density material within the fissure is talc from a prior pleurodesis. (B) 2011: CT image shows extensive mass involving the left pleural space extending into the fissure, showing progressive disease. The mass is hypodense to adjacent chest wall musculature. Note the subtle very fine internal separations within the mass lesion (red arrow). The high density within the fissure is talc from a prior pleurodesis (green arrow). (C) 2015: Stable disease compared with 2011. 


\section{Pathologic Findings}

Pathologic examination of multiple sites, including the spleen in 2002, supraclavicular lymph node in 2008, and the left lower lobectomy specimen and pleural rind in 2012, all demonstrated proliferation of lymphatic channels. The left lower lobectomy showed diffuse proliferation of lymphatics accentuated around bronchovascular bundles and visceral pleura (Figure 2). Immunostain for D2-40, a lymphatic endothelial marker, ${ }^{3}$ was positive (Figure 2A). Evidence of a prior pulmonary hemorrhage consisting of intra-alveolar hemosiderin-laden macrophages was present. Additionally, there was interstitial fibrosis with prominent encrustation of the elastic fibers in the alveolar septa and vessels with iron and calcium and associated giant cell reaction (Figure 2C, D). Given the diffuse proliferation of the lymphatics in multiple organs, a diagnosis of lymphangiomatosis (GLA) was made.

Molecular testing for EGFR and KRAS mutations and ALK rearrangement was negative. Immunostain for c-Kit was positive only in the lymphatic endothelial cells (Figure 2B). Targeted next-generation gene sequencing with a 50-gene panel (AmpliSeq Hotspot Panel; Life Technologies, Inc., Grand Island, NY) showed that the patient harbored the KIT M541L variant in both the lymphangiomatosis and normal lung, suggesting the possibility of germline

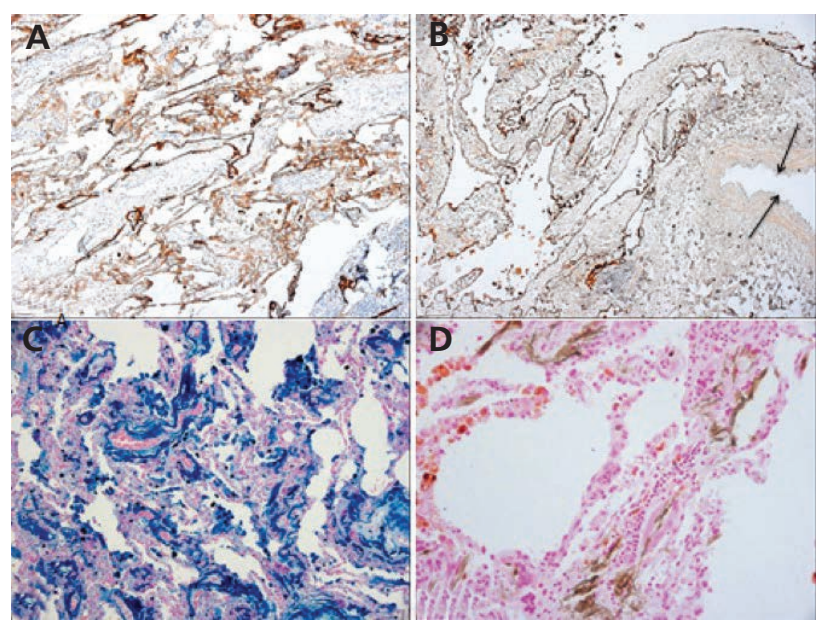

Figure 2. Surgical pathology. (A) Immunostain for D2-40 highlights the dilated lymphatics in the lung parenchyma (original magnification $x 10)$. (B) Immunostain for C-KIT is positive in the endothelial cells of lymphangiomatosis, and endothelium of an adjacent artery (arrows) is negative (original magnification $\mathrm{x} 4$ ). The adjacent lung shows heavy encrustation with (C) iron (Prussian blue, original magnification $\times 10$ ) and (D) calcium (von Kossa, original magnification x20). mutation. The presence of a germline mutation was confirmed by testing DNA extracted from nucleated cells in the patient's blood.

\section{Discussion}

Precision medicine seeks genetic mutations or biomarkers that lead to specific therapy for an individual or disease. ${ }^{4}$ Based on overexpression of VEGR receptors (VEGFRs) and tyrosine kinases in other vascular endothelial cell tumors, such as angiosarcoma $^{5}$ and lymphangioma, ${ }^{6-8}$ and due to the lack of effective therapy in lymphangioma, antiangiogenic therapy seemed a reasonable therapy in this patient with lymphangiomatosis, a condition with abnormal proliferation of lymphatic endothelial cells. Therefore, sorafenib was prescribed, which is a small molecule inhibitor of several tyrosine kinases (ie, VEGFR, platelet-derived growth factor receptor [PDGFR], and Raf kinases) and approved for the treatment of advanced renal cell carcinoma, hepatocellular carcinoma (HCC), and thyroid carcinoma. ${ }^{9}$ However, it was discontinued because of skin toxicity and lack of therapeutic effect (persistent chest pain, dyspnea, and hemoptysis). Similarly, sirolimus, an mTOR inhibitor known to reduce lymphatic endothelial cell growth, ${ }^{10}$ was prescribed but resulted in no success. Orbital lymphangioma in children is positive for PDGF alpha and beta receptors in the lymphatic endothelium, and c-KIT was expressed in mast cells. ${ }^{11}$ In our patient, lymphatic endothelial cells displayed positive staining for $\mathrm{c}-\mathrm{KIT}$, suggesting a possible role in the progressive lymphatic proliferation. CD117 (c-KIT) antibody is a stem cell factor receptor that inhibits apoptotic cell death upon binding of its ligand. Imatinib, a c-KIT inhibitor, was therefore initiated and resulted in clinical improvement and regression of disease over the past 4 years. To date, the patient has experienced no further hemoptysis, hemothorax, chest pain, or lymphadenopathy, and lung function has improved.

Upregulation of tyrosine kinase, as suggested by the positive immunostaining for $\mathrm{c}-\mathrm{KIT}$, is an important driver of neoplasia. A diverse variety of tumors have been found to contain this marker, including chronic myelogenous leukemia (CML), gastrointestinal stromal tumors (GISTs), angiosarcoma, ovarian granulosa cell tumors, renal cell carcinoma, renal oncocytoma, anaplastic thyroid carcinoma, cholan- 
Libby et al

giocarcinoma, desmoid tumors, and HCC. Elevated expression of c-KIT has also been found in small venous malformations in the blue rubber bleb nevus syndrome. ${ }^{12}$

Mutations in KIT occur in germline or somatic configuration. Germline mutations of exons 11, 13, and 17 of the KIT gene are frequently reported in familial and multiple GIST, ${ }^{13}$ familial mastocytosis, ${ }^{14}$ and aggressive fibromatosis. ${ }^{15}$ Multiple activating KIT mutations in exons 9, 11, 13, and 17 have been described in GIST, melanoma, acute myeloid leukemia, and systemic mastocytosis. ${ }^{16-18}$ The M541L variant that was present in our patient on exon 10 of KIT has been associated with several neoplastic disorders, occurring with higher frequency in CML, dedifferentiated liposarcomas, and pediatric mastocytosis. ${ }^{19}$ In vitro studies have shown that the tyrosine kinase activation of murine KIT L540 increased compared with KIT wild-type. ${ }^{20}$

Dufresne et $\mathrm{al}^{15}$ reported increased sensitivity to imatinib in patients with aggressive fibromatosis harboring the M541L variant of KIT, and all 3 patients with the M541L variant treated with imatinib achieved tumor control, including one patient who experienced complete response. In another study, low-dose imatinib was shown to be effective in a subset of patients with chronic eosinophilic leukemia. ${ }^{21}$ Although the disease associations with the KIT M541L variant need further investigation, its utility as a target is interesting.

Genetic analysis may be useful in rare nonmalignant disorders for which large clinical trials are not possible, an approach used for our patient with lifethreatening pulmonary lymphangiomatosis. To our knowledge, this is the first report in which positive staining of lymphatic endothelial cells for c-KIT and the finding of an M541L variant of a KIT germline mutation led to targeted treatment of lymphangiomatosis with imatinib, resulting in durable clinical improvement. Our patient has remained clinically stable on imatinib for 4 years, and we anticipate that she will be continued on it as long as she experiences response. The clinical benefit she has received from imatinib is cessation of recurrent hemoptysis which was not accompanied by significant anatomic shrinkage by imaging, perhaps through stabilization of proliferation of lymphatic endothelium. The biologic effect of imatinib, when prescribed at higher concentrations, has been shown to inhibit angiogenesis by blocking the activity of c-kit tyrosine kinase cell surface receptor and PDGFR kinase, ${ }^{22}$ and through this mechanism, is perhaps preventing further hemorrhage in our patient.

\section{References}

1. Lim H, Han JH, Kim HK, Kim TS. A rare case of diffuse pulmonary lymphangiomatosis in a middle-aged woman. Korean J Radiol 2014;15:295299.

2. Choi SH, Kim L, Lee $\mathrm{KH}$, et al. Mediastinal lymphangioma treated using endobronchial ultrasound-guided transbronchial needle aspiration. Respiration 2012;84:518-521.

3. Kahn HJ, Marks A. A new monoclonal antibody, D2-40, for detection of lymphatic invasion in primary tumors. Lab Invest 2002;82:1255-1257.

4. Collins FS, Varmus H. A new initiative on precision medicine. N Engl J Med 2015;372:793-795.

5. Styring E, Seinen J, Dominguez-Valentin M, et al. Key roles for MYC, KIT and RET signaling in secondary angiosarcoma. Br J Cancer 2014;15:407-412.

6. Sidle DM, Maddalozzo J, Meier JD, et al. Altered pigment epitheliumderived factor and vascular endothelial growth factor levels in lymphangioma pathogenesis and clinical recurrence. Arch Otolaryngol Head Neck Surg 2005;131:990-995.

7. Huang HY, Ho CC, Huang PH, Hsu SM. Co-expression of VEF-C and its receptors, VEGFR-2 and VEGFR-3, in endothelial cells of lymphangioma. Implication in autocrine or paracrine regulation of lymphangioma. Lab Invest 2001;81:1729-1734.

8. Itakura E, Yamamoto H, Oda Y, et al. VEGF-C and VEGFR-3 in a series of lymphangiomas: is superficial lymphangioma a true lymphangioma? Virchows Arch 2009;454:317-325.

9. Gotink KJ. Anti-angiogenic tyrosine kinase inhibitors: what is their mechanism of action? Angiogenesis 2010;13:1-14.

10. Bissler JJ, McCormack FX, Young LR, et al. Sirolimus for angiomyolipoma in tuberous sclerosis complex or lymphangioleiomyomatosis. N Engl J Med 2008;358:140-151.

11. Esmaeli B, Prieto VG, Gonnering R, et al. Growth factor receptor expression in orbital lymphangioma: possible therapeutic implications. Orbit 2004;23:263269.

12. Mogler C, Beck C, Kulozik A, et al. Elevated expression of c-kit in small venous malformations of blue rubber bleb nevus syndrome. Rare Tumors 2010;2:e36.

13. Curtin JA, Busam K, Pinkel D, Bastian BC. Somatic activation of KIT in distinct subtypes of melanoma. J Clin Oncol 2006;24:4340-4346.

14. Patnaik MM, Rindos M, Kouides PA, et al. Systemic mastocytosis: a concise clinical and laboratory review. Arch Pathol Lab Med 2007;131:784-791.

15. Dufresne A, Bertucci F, Penel N, et al. Identification of biological factors predictive of response to imatinib mesylate in aggressive fibromatosis. $\mathrm{Br} \mathrm{J}$ Cancer 2010;103:482-485.

16. Corless CL, Heinrich MD. Molecular pathobiology of gastrointestinal stromal sarcomas. Annu Rev Pathol 2008;3:557-586.

17. Beadling C, Jacobson-Dunlop E, Hodi FS, et al. KIT gene mutations and copy number in melanoma subtypes. Clin Cancer Res 2008;1:6821-6828.

18. Orfao A, Garcia-Montero AC, Sanchez L, Escribano L. Recent advances in the understanding of mastocytosis: the role of KIT mutations. Br J Haematol 2007;138:12-30.

19. Krasagakis K, Metaxari M, Zervou M, et al. Identification of the M541L sequence variation of the transmembrane KIT domain in Merkel cell carcinoma. Anticancer Res 2011;31:807-811.

20. Inokuchi $K$, Yamaguchi $H$, Tarusawa $M$, et al. Abnormality of c-kit oncoprotein in certain patients with chronic myelogenous leukaemia - potential clinical significance. Leukemia 2002;16:170-177.

21. Lurlo A, Gianelli U, Beghini A, et al. Identification of KIT M541L somatic mutation in chronic eosinophilic leukemia, not otherwise specified and its implication in low-dose imatinib response. Oncotarget 2014;5:4665-4670.

22. Arora A, Scholar EM. Role of tyrosine kinase inhibitors in cancer therapy. J Pharmacol Exp Ther 2005;315:971-979. 